\title{
A Practical Framework for Virtual Viewing and Relighting
}

\author{
Qi Duan, Jianjun Yu, Xubo Yang, and Shuangjiu Xiao \\ Digital Art Laboratory, \\ School of Software \\ Shanghai Jiao Tong University \\ \{duanqi1983, jianjun-yu\}@sjtu.edu.cn, \{yangxubo, xsjiu99\}@cs.sjtu.edu.cn
}

\begin{abstract}
Recently many practical applications have concerned with observing objects that have specular reflection properties. They intend to know how the specular reflections and other details vary according to different lighting conditions and view positions. In this paper, we propose an innovative framework combining the novel view synthesis algorithm and the relighting algorithm to fulfill these requirements. Another important feature of this framework is that all the algorithms are based on image without acquiring any $3 \mathrm{D}$ model which may contain some high confidential secrets. Meanwhile an image measurement criterion is proposed to verify the feasibility and effectiveness of the framework.
\end{abstract}

Keywords: Novel View Synthesis, Image-based Relighting, Image Measurement.

\section{Introduction}

Recently, the technique that can render images of products under arbitrary illuminations and view points became increasingly important in movie, museums, and electronic commerce applications. Various special lights will be placed in certain positions to augment the effects of details on product surface; meanwhile the observation position and angle also influence the display result. The position of viewpoints and the condition of luminance are two key factors that influence the effects. To determine the best luminance condition and view point position, it is very helpful to design a tool that can precisely observe the target with specular propertities under various light conditions and in some arbitrary view positions. Considering that the product may contain some highly confidential secrets, those requirements can be summarized as follows:

- A method to simulate the result of the objects under various light conditions, for example, different numbers, sizes, types, colors of light resources and different distances between objects and light. And we need to get the best result with the minimal cost.

- A technique to synthesize novel images of the objects. Considering the objects may relate to commercial confidential secrets, the less $3 \mathrm{D}$ information of the target we calculate, the better. 


\subsection{Related Work}

Two main algorithms employed in this framework are the image-based relighting and the novel view synthesis.

Image-Based Relighting(IBL) attracts a lot of attention recently. It can be classified into three categories: reflectance function based relighting, basis function-based relighting and plenoptic function-based relighting. Reflectance Function-based Relighting techniques explicitly estimate the reflectance function at each visible point of the object, which is known as the Anisotropic Reflection Model[1] or the BSSRDF 2]. Basis Function-based Relighting techniques take advantage of the linearity of the rendering operator with respect to illumination. Relighting process is accomplished via linear combination of a set of pre-rendered "basis" images. Nimeroff et al. 3 used a technique of combining images to relight a scene. Debevec et al.4 describes a Light Stage in which an object can be placed. Plenoptic Function-based Relighting techniques are based on the computational model-the Plenoptic Function [5]. It extracts out the illumination component from the aggregate time parameter, and facilitate relighting of scenes.

Novel image synthesis has been studied in the last decade. The work can be divided into two main classes roughly: image-based modeling and rendering(IBMR) and image-based morphing and rendering. The first class is designed to reconstruct 3D models from photographs. Debevec et al. [6 have shown a method of modeling and rendering architecture from a small number of photos. Criminisi et al. [7] create 3D texture-mapped models relying solely on one single image as its input. Byong Mok Oh et al. 8 take a single photo as input and model the scene. The second class does not calculate any 3D information of the scene. It use various methods to synthesis the novel image such as image warping [9], image interpolation[10], image extrapolation and so on. For example, Seitz and Dyer 11] interpolate along the base line of image pairs to obtain novel images. Levoy and Hanrahan[12] and Gortler et al.13] interpolate between a dense set of several thousand-example images to obtain novel view images. Peleg and Herman [14] relax the fixed camera constraint to synthesis results. Avidan and Shashua 16 introduce the concept of trilinear tensor space and use trilinear tensor to synthesize the novel view images.

However, the image-base relighting approach and novel view synthesis method can only satisfy one of the two requirements. Our framework integrates the two algorithms closely, which can render images of products under arbitrary illuminations and view points.

In the following section 2 we introduce the framework in detail. Image measurement criterion and framework performance are described in section 3. Finally, we conclude and present the idea for future work in section 4 .

\section{Algorithms}

\subsection{Framework Architecture}

The most significant feature of the framework is that it can satisfy the specified requirements and is totally based on images. The framework integrates two 
important algorithms above as two modules. The structure is shown in Figure [1. Firstly in image-based relighting module, the camera position is fixed and the relighting process is performed, then the result image is obtained under arbitrary luminance situation as the "source image". After that the camera position and orientation are moved in a little range and a picture of the object is captured as the "reference image". Using source image and reference image as the input of novel view synthesis module, the final novel image can be synthesized. Once the light environment changes, the relighting result is recalculated and the source image is updated, leaving reference image unmodified. Then the novel image is synthesized again. Employing this framework, we can observe the target of various luminance situations under arbitrary viewpoints.

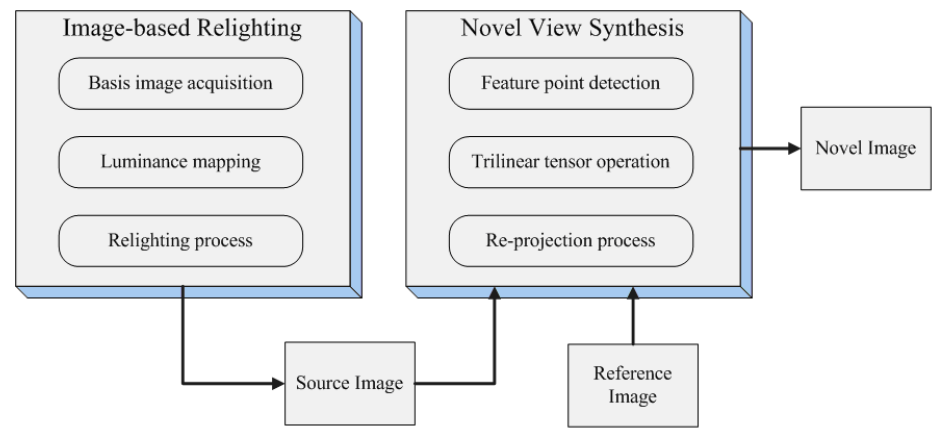

Fig. 1. Framework Architecture.The architecture contains two main algorithm modules. The source image is the output of relighting module, and the input of the second module, as well as the reference image. The novel image is the final result as the output of the novel module.

\subsection{Image-Based Relighting}

Our image-based Relighting (IBL) can synthesize realistic images without a complex and long rendering process as in traditional geometry-based computer graphics. The algorithm detail is divided into three parts-basis image acquisition,luminance mapping and relighting process.

To acquire the basis image,we design a spherical light stage with prepared "standard" point light source as "directional light source" attached on it. The light moves around the surface of sphere and for each light position and a corresponding basis image of objects placed at the center is captured. This process is repeated until the entire sphere of incident illumination is sampled at a predefined resolution.

To accelerate our whole process, we map the sphere map to a latitude-longitude map. Each region in the map has different scalaring factors because it has different impact on the final result, which can be determined by the following equation 1 .

$$
A_{i}=\frac{1}{4 \pi} \int_{\left(\phi_{i}-\frac{\Delta \phi}{2}\right)}^{\left(\phi_{i}+\frac{\Delta \phi}{2}\right)} \int_{\left(\phi_{i}-\frac{\Delta \theta}{2}\right)}^{\left(\phi_{i}+\frac{\Delta \theta}{2}\right)} \sin (\theta) d \theta d \phi
$$


To render the target under arbitrary illumination, the virtual light is mapped onto the sphere map first. Then the sphere map is converted to the Longitude-Latitude map. So the relighting problem can be solved by linear combination of the basis images.

\subsection{Novel View Synthesis}

The novel view synthesis algorithm is employed to synthesize the image under arbitrary view position. This technique is based on two or three sample images and the primary process is the tirlinear tensor operation, which is quite fit to our application.

To accelerate the process, we take two sample images, extract the putative match points [15] and then calculate a $3 \times 3 \times 3$ basis trilinear tensor matrix $T_{i}^{j l}[16$ ]. To acquire novel image of the object, a $3 \times 3$ rotation homography $R$ and a $3 \times 1$ translation vector $t$ should be specified,and the new tensor $G_{i}^{j k}$ will be calculated in equation 2 16]:

$$
G_{i}^{j k}=R_{l}^{k} T_{i}^{j l}+t^{k} a_{i}^{j}
$$

Using new tensor and sample images, the new novel image can be rendered.

\section{$3 \quad$ Experiments}

Regarding to the two different algorithms, diverse verification experiments are carried out separately according to different modules in this section.

To verify the accuracy of this relighting algorithm, we design a new approach that compare the histograms of the $R, G, B$ channel as well as the luminance channel. In addition, the mean value and variance of the two images are calculated. Here we show the analysis results of a example relighting result and the real image in Table 1. The range of error between image pair is limited to a very small fraction. This experiment proves the method used for image-based relighting is extremely accurate and efficient.

Table 1. Statistical result of the image pair

\begin{tabular}{|c|c|c|c|c|c|}
\hline \multirow{2}{*}{ Channel } & \multicolumn{2}{|c|}{ Mean Value } & \multicolumn{2}{c|}{ Variance } & \multirow{2}{*}{ Error } \\
\cline { 2 - 5 } & Our Method & Real Photo & Our Method & Real Photo & \\
\hline Red & 127 & 128 & 89.16 & 93.29 & $0.03 \%$ \\
\hline Green & 115 & 115 & 89.08 & 94.54 & $0.03 \%$ \\
\hline Blue & 120 & 122 & 90.81 & 95.77 & $0.02 \%$ \\
\hline Luminance & 119 & 119 & 81.94 & 88.04 & $0.02 \%$ \\
\hline
\end{tabular}

Considering the interpolation and extrapolation characteristic of novel view algorithm, the criterion is that the result satisfies people's vision effect and the physical distortions of the target object occur in an acceptable range. Figure 2 shows the experiment result of this module, which is also the final novel result of this framework. 


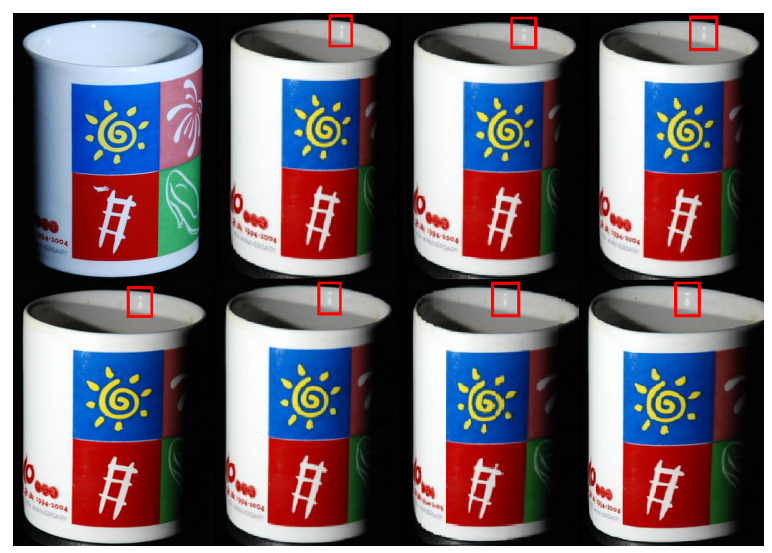

Fig. 2. Novel View Results. The left above image is the reference image and the left below image is the generated new source image. The other images are the synthesized novel view images that we specify the camera move around the Z-axis of the target from left to right.

\section{Conclusion and Future Work}

In this paper we present a framework to satisfy practical requirements of observing objects in various environments, especially the specular and shadow details on the surface. And we choose the image-based methods to avoid calculating 3D information or model. In the future, we will still do some researches in these aspects: accelerating the process of relighting, improving the accuracy of novel view methods, extending the novel view algorithm that can observe the target globally.

Acknowledgments. This research is sponsored by 863 National High Technology R\&D Program of China (No. 2006AA01Z307), National Natural Science Foundation of China (No. 60403044) and partially sponsored by Omron company.

\section{References}

1. Kajiya, J.T.: Anisotropic reflection models. In: SIGGRAPH '85. Proceedings of the 12th annual conference on Computer graphics and interactive techniques, pp. 15-21. ACM Press, New York (1985)

2. Jensen, H.W., Marschner, S.R., Levoy, M., Hanrahan, P.: A practical model for subsurface light transport. In: SIGGRAPH '01. Proceedings of the 28th annual conference on Computer graphics and interactive techniques, pp. 511-518. ACM Press, New York (2001)

3. Nimeroff, J., Simoncelli, E., Dorsey, J.: Efficient rerendering of naturally illuminated environments. In: Eurographics Rendering Workshop 1994, Darmstadt, Germany. Springer, Heidelberg (1994) 
4. Debevec, P., Hawkins, T., Tchou, C., Duiker, H.-P., Sarokin, W., Sagar, M.: Acquiring the reflectance field of a human face. In: SIGGRAPH '00. Proceedings of the 27th annual conference on Computer graphics and interactive techniques, pp. 145-156. ACM Press/Addison-Wesley Publishing Co., New York (2000)

5. Adelson, E.H., Bergen, J.R.: The plenoptic function and the elements of early vision. Computational Models of Visual Processing, 3.20 (2001)

6. Debevec, P.E., Taylor, C.J., Malik, J.: Modeling and rendering architecture from photographs: A hybrid geometry and image-based approach. In: Proc. SIGGRAPH'96, pp. 11-20. ACM Press, New Orleans, LS, USA (1996)

7. Criminisi, A., Reid, I., Zisserman, A.: Single View Metrology. International Journal of Computer Vision 40, 123-148 (2000)

8. Oh, B.M., Chen, M., Dorsey, J., Durand, F.: Image-based modeling and photo editing. In: SIGGRAPH '01. Proceedings of the 28th annual conference on Computer graphics and interactive techniques, pp. 433-442. ACM Press, New York (2001)

9. Beier, T., Neely, S.: Feature-Based Image Metamorphosis. In: ACM SIGGRAPH Computer Graphics, vol. 26, pp. 35-42. ACM Press, New York (1992)

10. Seitz, S.M., Dyer, C.R.: Physically-Valid View Synthesis By Image Interpolation. In: IEEE Workshop on Representation of Visual Scenes, p. 18. IEEE Computer Society Press, Los Alamitos (1995)

11. Seitz, S.M., Dyer, C.R.: View Morphing. In: SIGGRAPH '96. Proceedings of the 23rd annual conference on Computer graphics and interactive techniques, pp. 21-30. ACM Press, New York (1996)

12. Levoy, M., Hanrahan, P.: Light field rendering. In: SIGGRAPH '96. Proceedings of the 23rd annual conference on Computer graphics and interactive techniques, pp. 31-42. ACM Press, New York (1996)

13. Gortler, S.J., Grzeszczuk, R., Szeliski, R., Cohen, M.F.: The lumigraph. In: SIGGRAPH '96. Proceedings of the 23rd annual conference on Computer graphics and interactive techniques, pp. 43-54. ACM Press, New York (1996)

14. Peleg, S., Herman, J.: Panoramic Mosaic by Manifold Projection. In: IEEE Computer Society Conference on Computer Vision and Pattern Recognition, p. 338. IEEE Computer Society Press, Los Alamitos (1997)

15. http://www.cs.unc.edu/ blloyd/comp290-089/fmatrix/

16. Avidan, S., Shashua, A.: Novel View Synthesis by Cascading Trilinear Tensors. In: IEEE Transactions on Visualization and Computer Graphics (TVCG), vol. 04, pp. 293-306. IEEE Computer Society Press, Los Alamitos (1998) 